\title{
Identification of specific B cell linear epitopes of mycoplasma hyorhinis P37 protein using monoclonal antibodies against baculovirus-expressed P37 protein
}

\author{
Hongzhen Zhu ${ }^{1+}$, Yanwu Wei ${ }^{1 \dagger}$, Liping Huang ${ }^{1}$, Dan Liu², Yongxing Xie', Deli Xia ${ }^{1}$, Haiqiao Bian ${ }^{1}$, Li Feng ${ }^{1}$ and \\ Changming Liu ${ }^{1 *}$
}

\begin{abstract}
Background: Mycoplasma hyorhinis (Mhr) is the etiologic agent of lameness and polyserositis in swine. P37 is a membrane protein of Mhr that may be an important immunogen and is a potential target for diagnostic development. However, there is little information concerning Mhr P37 protein epitopes. A precise analysis of the P37 protein epitopes should extend our understanding of the antigenic composition of the P37 protein and the humoral immune responses to Mhr infection. Investigating the epitopes of Mhr P37 will help to establish a detection method for Mhr in tissue and provide an effective tool for detecting Mhr infection.

Results: Western blot and indirect immunofluorescence assays (IFA) confirmed that the expressed P37 protein was recognized by Mhr-positive porcine and mouse sera. Furthermore, the P37 protein was purified using affinity chromatography and used to immunize mice for hybridoma cell fusion. Four monoclonal antibodies (mAbs) found to be positive for Mhr were detected in infected lung tissue. A panel of truncated P37 proteins was used to identify the minimal $B$ cell linear epitopes of the protein based on these mAbs. The core epitope was determined to be ${ }^{206}$ KIKKAWNDKDWNTFRNF ${ }^{222}$

Conclusions: In this study, we identified 17 critical amino acids that determine the epitope of the P37 protein of Mhr. This study identified mAbs that could provide useful tools for investigating the Mhr P37 antigenic core epitope (amino acids 206-222) and detecting Mhr-specific antigens in infected tissue.
\end{abstract}

Keywords: Mycoplasma hyorhinis, P37 protein, Monoclonal antibody, Antigenic epitope

\section{Background}

Mycoplasma hyorhinis (Mhr) was first isolated in 1953 and found to lack a cell wall [1]. It is a commensal microorganism that inhabits the upper respiratory tract of swine [2]. Mhr infections in pigs can cause lameness and polyserositis, and severe infections can cause pneumonia $[3,4]$. Systemic infection caused by Mhr is found on pig farms worldwide and is characterized by high

\footnotetext{
* Correspondence: liuchangming01@caas.cn

†Hongzhen Zhu and Yanwu Wei contributed equally to this work

'Division of Swine Digestive System Infectious Diseases, State Key Laboratory of Veterinary Biotechnology, Harbin Veterinary Research Institute, Chinese Academy of Agricultural Sciences, No. 678 Ha-ping Street, Xiang-fang region, Harbin 150069, China

Full list of author information is available at the end of the article
}

morbidity and low mortality rates $[5,6]$. At present, $\mathrm{Mhr}$ infection detection mainly depends on pathogen isolation and culture and polymerase chain reaction (PCR) methods; and there is no commercially available kit for serological detection, as Mhr is a commensal in the respiratory tract and tonsils of pigs, presence of antibodies does not indicate $M$. hyorhinis as an etiologic agent of clinical signs [2, 7]. Although Mhr is easily isolated from porcine alveolar lavage fluid and nasal swabs, the process of isolation and identification of Mhr is often time consuming [8]. Mhr has been proven to be a zoonotic pathogen and identified in co-infection with PRRSV or PCV2 in the porcine respiratory system [9-12]. In

(C) The Author(s). 2019 Open Access This article is distributed under the terms of the Creative Commons Attribution 4.0 International License (http://creativecommons.org/licenses/by/4.0/), which permits unrestricted use, distribution, and reproduction in any medium, provided you give appropriate credit to the original author(s) and the source, provide a link to the Creative Commons license, and indicate if changes were made. The Creative Commons Public Domain Dedication waiver (http://creativecommons.org/publicdomain/zero/1.0/) applies to the data made available in this article, unless otherwise stated. 
general, the treatment of Mhr infection is mainly through the use of antibiotics [5].

Members of the variable lipoprotein family of Mhr have been shown to perform a variety of adherence functions during infection and interactions with the host, which presumably facilitates chronic infections [13, 14]. P37 is an important membrane protein of $\mathrm{Mhr}$ and is part of the periplasmic binding protein-dependent transport system $[15,16]$. P37 may play a role in tumor invasion, and detection of antibodies against P37 in human serum may help diagnose cancer $[17,18]$.

Previously, the P37 protein was used as a coating antigen to measure the immunoglobulin $\mathrm{G}$ (IgG) responses in swine vaccinated with an inactivated Mhr vaccine [19]. However, it was unclear whether P37 protein could be used as an accurate indicator to identify naturally infected pigs in lung tissues, the role of P37 in the process of infection, or the precise epitope of P37.

In this study, we used monoclonal antibodies (mAbs) prepared in mice based on P37 protein expressed using the baculovirus expression system to detect the distribution of Mhr in infected tissues by immunohistochemistry and identify the core epitope of P37 protein using the truncated protein method.

\section{Results}

Identification of recombinant plasmid and shuttle plasmid The recombinant plasmid pFastBac ${ }^{\mathrm{Tw}} 1$-His-P37 was identified by dual-restriction endonuclease digestion with $\mathrm{BamH}$ I and Xho I, and the $4693 \mathrm{bp}$ vector fragment and the 1140 bp target gene fragment were visualized by $1 \%$ agarose gel electrophoresis (Fig. 1). pFastBac ${ }^{\text {ru }} 1$-His-P37 was specifically amplified using M13 primers, and a 3440 bp band was obtained on a $1 \%$ agarose gel. The negative control pFast$\mathrm{Bac}^{\mathrm{Tx}} 1$ was observed as a $2300 \mathrm{bp}$ fragment (Fig. 1).

\section{Detection and purification of recombinant protein}

Using fluorescence microscopy, strong fluorescence was observed in insect cells infected with pFastBac ${ }^{\text {Tx}} 1$-HisP37, whereas no fluorescence was observed in uninfected cells (Fig. 2), indicating that the P37 protein was successfully expressed in insect cells. Western blot analysis showed that the protein reacted with the prepared antiMhr mouse positive serum, and a specific reaction band appeared at approximately $43.3 \mathrm{kDa}$ (Fig. 3).

\section{Characterization of $\mathrm{P} 37$ protein-specific mAbs}

The four positive mAbs were designated C6, C8, E1, and E6. Subtype identification results showed that the heavy chain subtype of all four mAbs was IgG1, and the light chain subtype of all mAbs was kappa (Table 1). Reactivity analysis of mAbs to Mhr showed that the Mhr strain reacted specifically with the four mAbs, and a specific reaction band appeared at a position of $43.3 \mathrm{kDa}$ (Fig. 4). Immunohistochemical detection showed that Mhr-specific antigen was present in the lungs of confirmed Mhr-positive cases, and Mhr-specific antigen mainly localized to epithelial cells of lung tissues, with no Mhr-specific antigen and Mhp-specific antigen observed in uninfected lung tissue and Mhp positive lung tissue (Fig. 4).

\section{Identification of B cell linear epitopes of P37 using P37- specific mAbs}

To determine the epitopes of the four generated mAbs, a series of overlapping peptides were analyzed by Western blot analysis. The results showed the presence of specific bands at amino acids (aa) 128-254, aa171-254, and aa199-226 (Fig. 5). Because the four mAbs were identical in reactivity, only one result is shown here.

To confirm the epitopes of the mAbs, Western blot analysis was performed after mutating each aa one by one. No specific bands were detected after deletion of aa ${ }^{206}$ Lys (Fig. 6a) and aa ${ }^{222}$ Phe (Fig. 6b). This indicates that aa ${ }^{206}$ Lys and aa ${ }^{222}$ Phe are key amino acids of the core epitope of the P37 protein.

\section{Analysis of P37 protein from different Mhr strains}

Analysis of the p37 sequence of seven Mhr strains demonstrated that the epitope ${ }^{206}$ KIKKAWNDKDWNTFRNF ${ }^{222}$ was highly conserved (100\% aa identity, Fig. 7).

\section{Homology modeling of the P37 protein epitope}

The spatial structure of the P37 protein from aa1-379 was predicted using three-dimensional homology modeling. Model analysis showed that the overall shape of P37 is an irregular prolate ellipsoid, and the core epitope domain consists of two $\alpha$-helices and a nonregular coil (Fig. 8a and b). The epitope region where the antigenantibody reaction was detected was located on the surface of the P37 protein (Fig. 8c).

\section{Discussion}

A previous study suggested that Mhr was present in serum samples from pigs at fattening farms [20]. Although most pigs infected with Mhr do not show significant clinical symptoms, typical lesions found in infected 1-day to 1-year-old pigs include serum fibrotic pleurisy, otitis media, pericarditis and peritonitis, which may cause fibrous adhesions during the chronic phase [21-23]. Hence, accurate diagnosis is critical to establish effective treatment measures in affected herds.

P37 is thought to function in adherence to host cells and has been used as an antigen to monitor the production of antibodies in animals immunized with inactivated $\mathrm{Mhr}$ vaccine $[17,19,24]$. In this study, we focused on the conserved P37 protein of Mhr as a potential target for diagnostic development. We used mAbs prepared in mice based on P37 

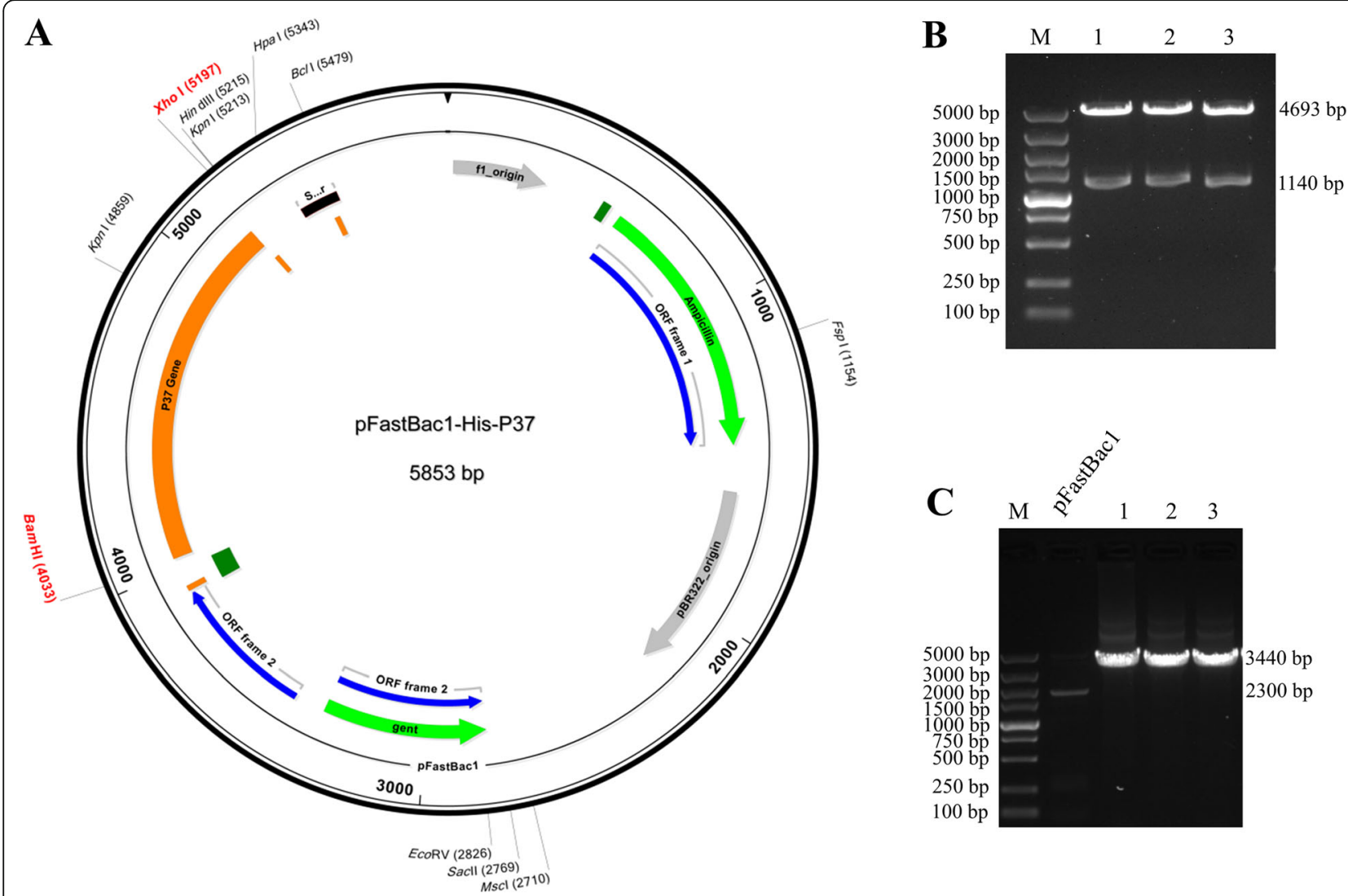

Fig. 1 Identification of recombinant plasmid and shuttle plasmid. a The restriction map and primer-binding sites. The P37 gene (1140 bp) was subsequently cloned into the expression vector pFastBac ${ }^{\mathrm{TM}} 1$ via two restriction sites (BamH I and Xho I). b Double enzyme digestion (BamH I and Xho I) revealed specific bands at $4693 \mathrm{bp}$ and $1140 \mathrm{bp}$. Lane M indicates the DNA molecular quality standard. Lanes 1, 2, and 3 represent plasmids extracted after single-colony expansion of randomly selected white colonies based on the Bac-to-Bac ${ }^{\circledast}$ Baculovirus Expression System instruction Version D (Invitrogen, Carlsbad, CA, USA). c The pFastBac ${ }^{\top \mathrm{M}}$ 1-His-P37 shuttle plasmid was identified using M13 primers, and a specific band appeared at $3440 \mathrm{bp}$, whereas pFastBac1 showed a specific band at $2300 \mathrm{bp}$. Lane M indicates the DNA molecular quality standard. Lane pFastBac1 indicates the pFastBac ${ }^{\mathrm{TM}} 1$ plasmid negative control. Lanes 1,2, and 3 represent plasmids extracted after single-colony expansion of randomly selected white colonies as described above

A

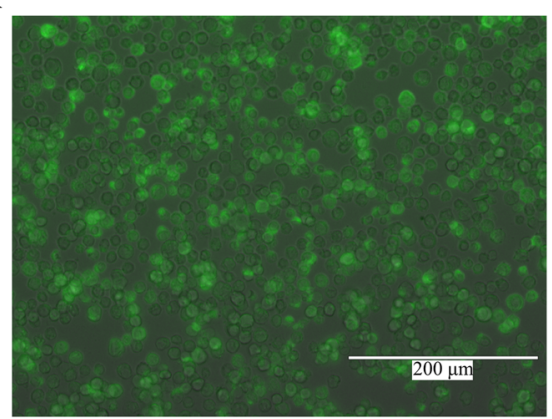

B

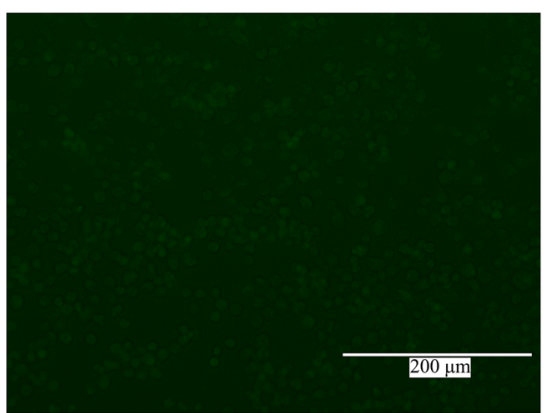

Fig. 2 Detection of recombinant P37 protein by indirect immunofluorescence assay (IFA). a Detection of Sf21 cells infected with pFastBac ${ }^{\text {TM}} 1$-HisP37 by IFA. Infected insect cells showed strong fluorescence. $\mathbf{b}$ Detection of uninfected Sf21 cells by IFA. No fluorescence was detected from negative cells 


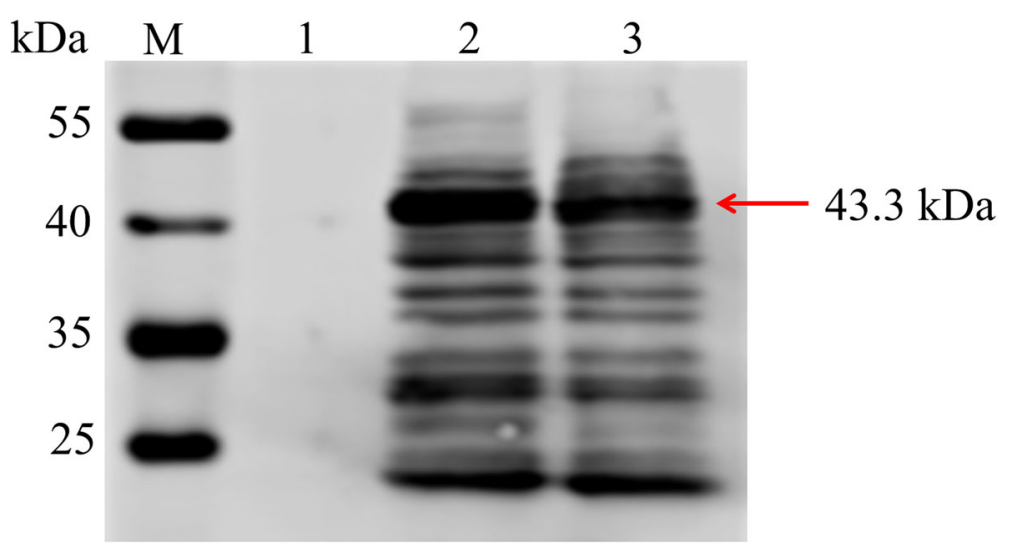

Fig. 3 P37 protein identified by Western blot analysis. Lane M, PageRuler ${ }^{\text {TM }}$ Prestained Protein Ladder (Thermo, Vilnius, Lithuania); lane 1, cell lysate from normal Sf21 cells; lane 2, recombinant P37 protein expressed in the baculovirus expression system; lane 3, recombinant P37 protein purified from the baculovirus expression system

protein expressed using the baculovirus expression system to establish an immunohistochemical method, which can be used to detect Mhr-specific antigen in naturally infected tissues. A precise analysis of P37 protein epitopes should extend our understanding of the antigenic composition of the P37 protein.

In this study, four P37-specific mAbs were produced using His-fused P37 expressed in sf21 cells as the immunogen. A series of GST-fused partial regions of P37 expressed in Escherichia coli were used to identify linear B cell epitopes in the P37 protein. Western blotting analysis showed that the four antibodies identified the same epitope, ${ }^{206} K_{\text {KIKKAWNDKDWNTFRNF }}{ }^{222}$. We speculated that the P37 protein expressed in sf21 cells was modified compared to that expressed in E. coli, which is consistent with previously described results [18]. P37 protein expressed in sf21 cells has post-translational modifications compared to P37 protein expressed in E. coli. However, the mechanism of P37 protein modification in sf 21 cells is unknown and requires further study.

Based on alignment of P37 amino acids, sequence analysis of different Mhr strains showed that the ${ }^{206} \mathrm{KIKK}$ AWNDKDWNTFRNF ${ }^{22}$ epitope was completely conserved in all Mhr strains. Although antigenic epitopes are usually contained within aa6-10, interestingly this epitope region was in aa17 $[25,26]$. Structural predictions by $3 D$ homology modeling revealed that the epitope is exposed on the surface of the P37 protein and includes a non-regular

Table 1 Identification of subclasses of mAbs in hybridoma cell supernatants

\begin{tabular}{|c|c|c|c|c|}
\hline & \multicolumn{4}{|c|}{ Hybridoma } \\
\hline & $\mathrm{C} 6$ & $\mathrm{C} 8$ & E1 & E6 \\
\hline Ig subclass & $\lg G 1$ & $\lg G 1$ & $\operatorname{lgG} 1$ & $\lg G 1$ \\
\hline Light chain type & K & K & K & K \\
\hline
\end{tabular}

coil. The fact that the functional part of the enzyme was often in the conformational region of the non-regular coil indicates that the establishment of an epitope-based diagnostic method could potentially exploit antigens for $\mathrm{Mhr}$ detection and avoid cross-reactions with Mycoplasma hyopneumoniae (Mhp) [27].

Immunohistochemical analysis showed that mAbs against the P37 protein can be applied to detect Mhr antigen in the lung tissue of Mhr-infected animals. Mhr mainly infects epithelial cells in the lungs, which is consistent with previously described results [28]. Therefore, we believe that mAbs against P37 protein expressed in sf 21 cells could be used to detect Mhr infection in pigs.

\section{Conclusions}

This study identified the core epitope of the P37 protein as ${ }^{206}$ KIKKAWNDKDWNTFRNF ${ }^{222}$. Homology modeling analysis showed that this epitope consists of two $\alpha$ helices and a non-regular coil. Immunohistochemistry results indicated that the epitope of the P37 protein may be involved in the production of host antibodies.

\section{Methods}

Pathogens, plasmids, cells, and anti-Mhr serum

The previously described Mhr-DL strain (CGMCC No. 11092) was used as the coating antigen for screening and Western blot analysis of mAbs [29]. Anti-Mhr mouse serum was prepared using the Mhr-DL strain as an immunogen. The baculovirus transfer vector pFast$\mathrm{Bac}^{\mathrm{TM}} 1$ (Invitrogen, Carlsbad, CA, USA) was used to construct the pFastBac ${ }^{\mathrm{rm}} 1$-His-p37 plasmid, which expressed the complete P37 protein. The prokaryotic expression vector pGEX-6P-1 (GE Healthcare, Uppsala, Sweden) was used to express the truncated P37 protein. The insect cell line Spodoptera frugiperda (sf21) (Invitrogen, Carlsbad, CA, USA) was cultured in Grace's Insect 
$\mathbf{A}$

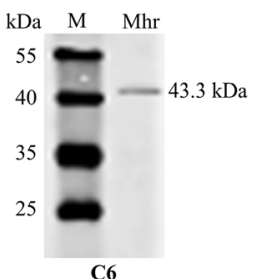

B

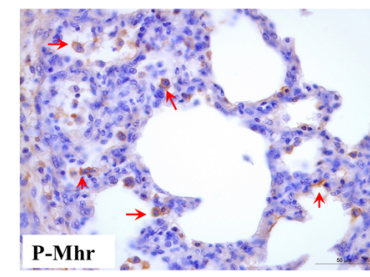

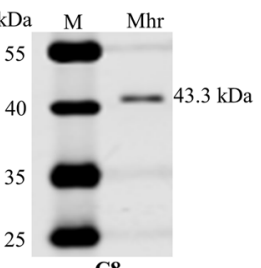

C8

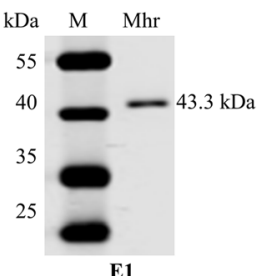

E1

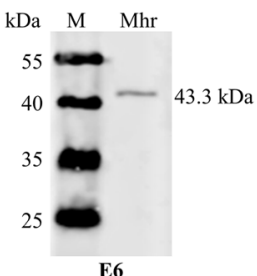

E6

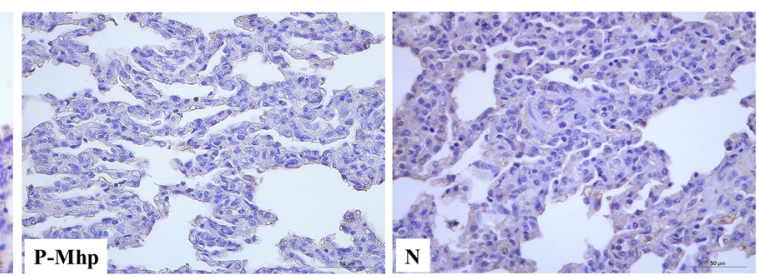

Fig. 4 Western blot analysis of four mAbs to Mhr. a Western blot analysis revealed four mAbs (C6, C8, E1, and E6). A specific Mhr band was detected at $43.3 \mathrm{kDa}$, indicating that the P37 protein expressed by the eukaryotic expression system had good immunogenicity. Lane M, PageRuler ${ }^{\text {TM }}$ Prestained Protein Ladder; lane Mhr, Mhr lysate. $\mathbf{b}$ Immunohistochemical analysis of Mhr-specific antigens in infected lung tissue using prepared mAbs. Positive signals (red arrows) were detected for the Mhr-specific antigen in infected lung tissue (P-Mhr) but were not detected in Mhp positive lung tissue (P-Mhp) and uninfected lung tissue (N)

Medium (Invitrogen) at $27^{\circ} \mathrm{C}$ to propagate recombinant baculovirus. The SP2/0 myeloma cell line (ATCC ${ }^{\circ} \mathrm{CRL}^{-}$ $1581^{\mathrm{Tx}}$ ) was cultured in Dulbecco's modified Eagle's medium (DMEM) (Invitrogen, Grand Island, NY, USA) with $10 \%$ inactivated fetal bovine serum (FBS, Thermo Fisher Scientific, North Shore City, New Zealand) in a humidified incubator with $5 \% \mathrm{CO}_{2}$ at $37^{\circ} \mathrm{C}$ and was then fused with mouse spleen cells.
The mouse anti-Mhr serum was prepared as follows: $\mathrm{M}$. hyrohinis $\left(1 \times 10^{10.0} \mathrm{CCU} / \mathrm{mL}\right)$ mixed to a concentration of $15 \%$ (v/v) with Seppic Montanide ISA15 VG (www.seppic.com), and then one 6-week-old female BALB/c mice were immunized with $500 \mu \mathrm{L}$. This immunization was repeated 3 weeks later and after further 2 weeks, mice were euthanized and collected blood, and then centrifugation $8000 \mathrm{rpm} / \mathrm{min}$ for $10 \mathrm{~min}$ at $4{ }^{\circ} \mathrm{C}$.

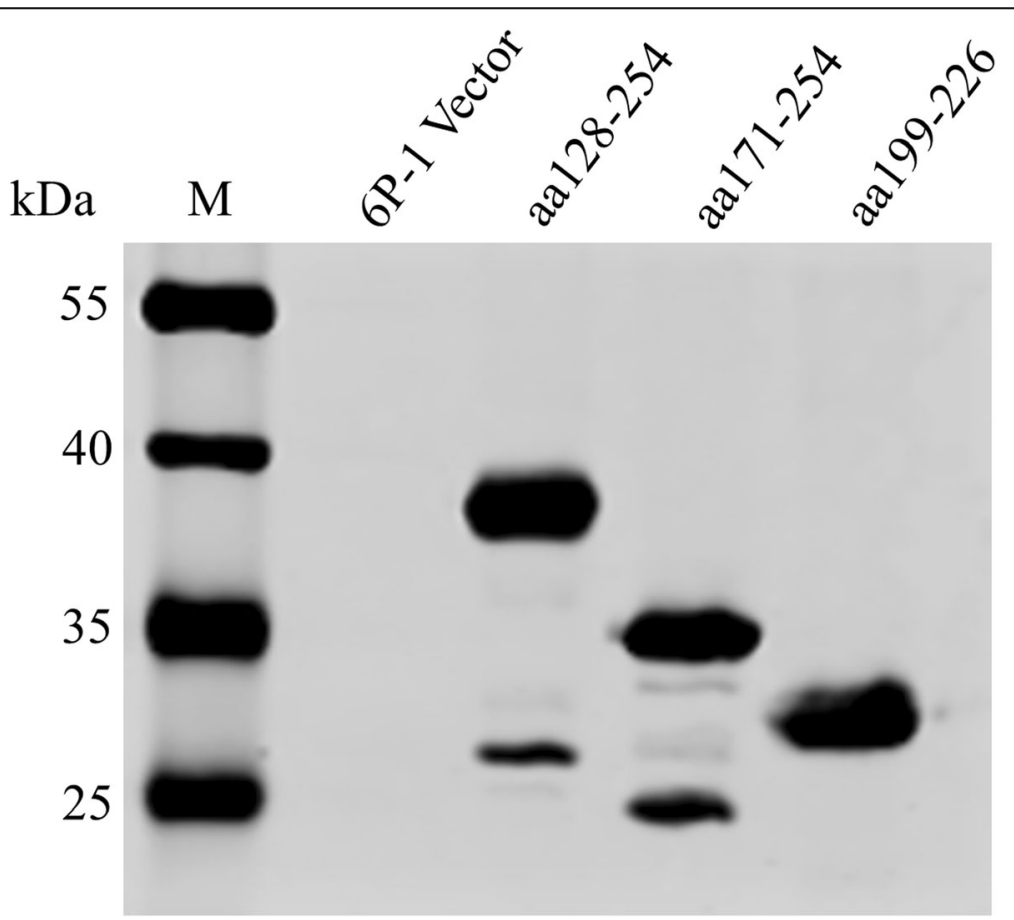

Fig. 5 Western blot analysis of mAbs that reacted specifically with the P37 protein fragment. Western blot analysis showed that mAbs reacted specifically with aa128-254, aa171-254, and aa199-226, and the results were consistent for the four mAbs. Lane M, marker; lane 6P-1, vector lysate after IPTG induction 

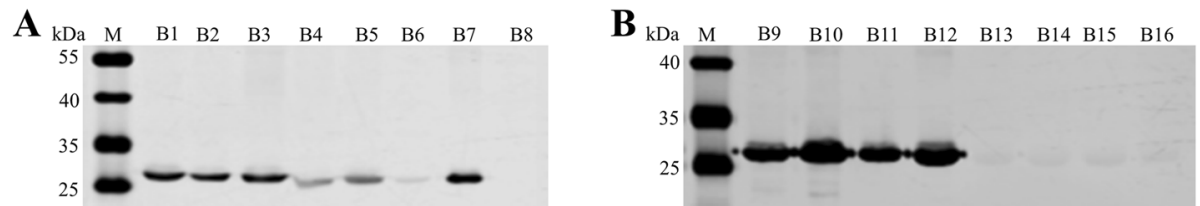

Fig. 6 Western blot analysis verification of key amino acids. In order to identify key amino acids, peptides were deleted one by one. B1-B8 indicates deleted peptides aa209-206, respectively. B9-B16 indicates deleted peptides aa226-219, respectively. a No specific band was detected when ${ }^{206}$ Lys was deleted. $\mathbf{b}$ No specific band was detected when ${ }^{222}$ Phe was deleted

\section{Acquisition of the p37 gene and generation of recombinant baculovirus}

The p37 gene fragment was amplified by PCR with forward (5' -CGGGATCCATGCTGAAGAAGCTGAAG-3') and reverse (5'-CCGCTCGAGTTACTTGATGGCCTTCTC-3') primers designed using Premier 5.0 software (PREMIER Biosoft, Palo Alto, CA, USA) based on the reference sequence (GenBank Accession No. X14140.1). The signal peptide sequence was removed from the p37 gene coding region and the p37 gene was optimized and synthesized by BGI Co. (Beijing, China). For protein purification, a $6 \times$ His-tag was fused to the $\mathrm{NH}_{2}$ terminal end of the p37 gene. The gene sequence was optimized to obtain the highest possible level of expression, and the target gene $(1140 \mathrm{bp})$ was subsequently cloned into the expression vector $\mathrm{pFastBac}^{\mathrm{\tau M}} 1$ via two restriction sites (BamH I and Xho I) (Fig. 1).

The linearized baculovirus DNA was transformed into competent DH10Bac (Invitrogen) according to the manufacturer's instructions. Identification of recombinant baculovirus was carried out using universal M13 primers (forward primer: 5'-GTTTTCCCAG TCACGAC-3', reverse primer: 5'-CAGGAAACAG CTATGAC-3'). pFastBac ${ }^{\mathrm{Tm}} 1$-His-P37 was transfected into logarithmic phase Sf21 insect cells according to the Bac-to-Bac ${ }^{\oplus}$ Baculovirus Expression System instructions (Invitrogen). When the cells exhibited obvious cytopathic effects, the cell supernatant was collected as the first generation recombinant baculovirus and designated P1 baculovirus. P1 baculovirus was then used to infect cells by performing a viral plaque assay to generate a high viral titer of P2 baculovirus. Prepared P2 baculovirus was used for expression studies.

\section{Expression and purification of P37 protein}

For P37 protein expression, three generations of high titer seed virus stocks were prepared by infecting Sf21 cells at a multiplicity of infection (MOI) of 0.1 plaque forming units (PFUs). Cells in 24-well plates were infected with pFastBac ${ }^{\mathrm{TM}} 1$-His-P37 baculovirus, and uninfected Sf 21 cells were used as mock control. The cell culture medium was aspirated after $60 \mathrm{~h}$ and fixed with $10 \%$ paraformaldehyde $(500 \mu \mathrm{L}$ per well $)$ at room temperature for $15 \mathrm{~min}$. The Sf21 cells were then fixed with $0.2 \%$ TritonX-100 for $10 \mathrm{~min}$, washed three times with phosphate buffered saline (PBS), and incubated with mouse anti-Mhr serum (Anti-Mhr serum was prepared and stored by our laboratory, and diluted 1:500 in PBS) at $37^{\circ} \mathrm{C}$ for $1 \mathrm{~h}$. The cells were then washed three times with PBS, incubated with DyLight 488 AffiniPure Goat Anti-Mouse IgG $(\mathrm{H}+\mathrm{L})$ (diluted 1:500 in PBS; Pierce, Rockford, IL, USA) at $37^{\circ} \mathrm{C}$ for $1 \mathrm{~h}$, and washed three times with PBS. The fluorescent signal was visualized with an EVOS inverted fluorescence microscope (Life Technologies, Carlsbad, CA, USA).

Sf21 cells infected with 5 MOI pFastBac ${ }^{\mathrm{Tm}} 1-\mathrm{His}-\mathrm{P} 37$ virus were harvested $72 \mathrm{~h}$ post infection. Cells were suspended in PBS (1\% of the original volume), lysed by ultrasonic lysis for $30 \mathrm{~min}$ (pulse on $3 \mathrm{~s}$, pulse off $5 \mathrm{~s}, 130$ watts), centrifuged at $9000 \mathrm{rpm}$ for $30 \mathrm{~min}$ at $4{ }^{\circ} \mathrm{C}$ to remove the precipitate, and purified using Ni-NTA affinity chromatography (Genscript, Nanjing, China). The purified sample was mixed with $5 \times$ loading buffer at a ratio of $4: 1$ and boiled for $10 \mathrm{~min}$. The expressed protein was separated by $12 \%$ sodium dodecyl sulfate-polyacrylamide gel electrophoresis (SDS-PAGE) and transferred to a nitrocellulose membrane. The membrane was blocked with $5 \%$ skim milk powder in PBS solution overnight at

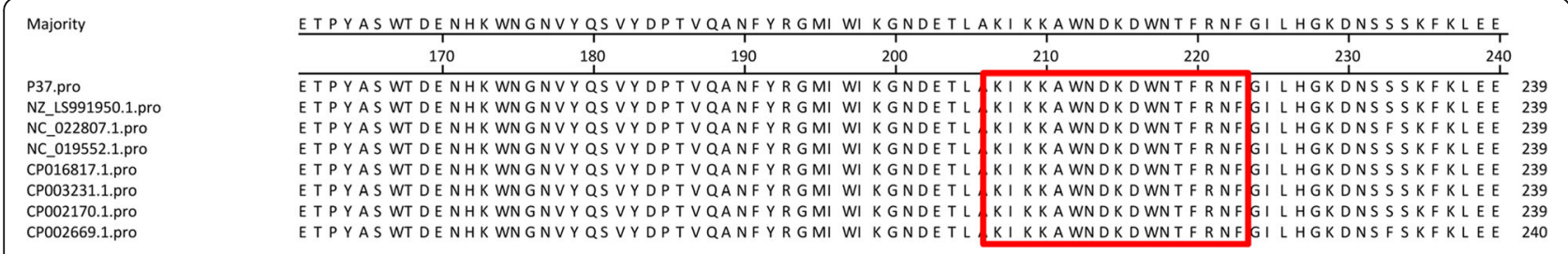

Fig. 7 Alignment of sequences with Mhr P37 epitopes. A total of seven Mhr strains were analyzed. The sequence motif recognized by mAbs is shown in the red box 

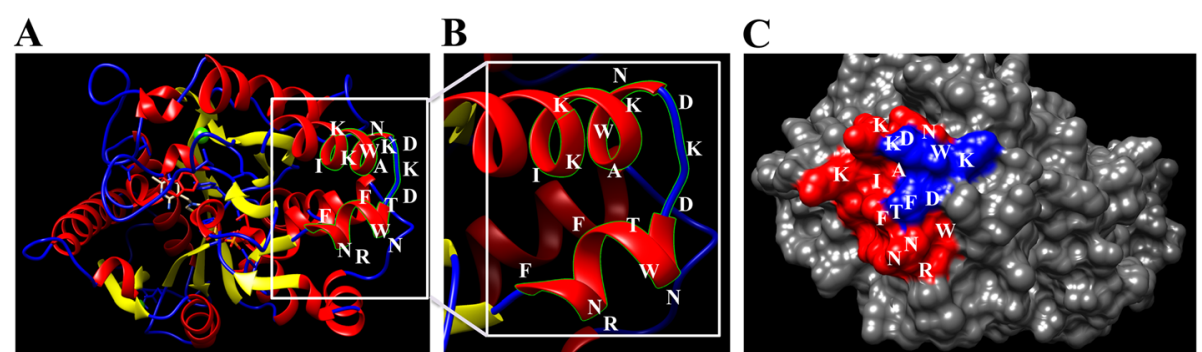

Fig. 8 Structural analysis of the P37 protein. Structural analysis and identification of B cell epitopes in the predicted three-dimensional structure of the P37 protein. Red areas represent a-helix regions, blue areas represent nonregular coil regions, and yellow areas represent strand regions. a The location of epitope ${ }^{206} \mathrm{KIIKAWNDKDWNTFRNF}^{222}$ is shown in a gray box. $\mathbf{b}$ Areas where antigen reacted to antibodies. $\mathbf{c}$ The epitope was located on the surface of the P37 protein

$4{ }^{\circ} \mathrm{C}$, incubated with anti-Mhr mouse serum $(1: 100)$ at room temperature for $1 \mathrm{~h}$, and washed three times with PBS containing $0.5 \%$ Tween 20 (PBST). After incubation for $1 \mathrm{~h}$ at room temperature with DyLight ${ }^{\mathrm{m}}$ 680-Labeled Antibody To Mouse IgG $(\mathrm{H}+\mathrm{L})$ (1:10000) (KPL, Gaithersburg, MD, USA), washed three times with PBST, and scanned using an Odyssey infrared imaging system (Licor Odyssey, Lincoln, NE, USA).

\section{Preparation of mAbs against $\mathrm{P} 37$ protein}

Four 6-week-old female BALB/c mice were purchased from the Laboratory Animal Center of Harbin Veterinary Research Institute, CAAS (Harbin, China). The mice were immunized with $80 \mu \mathrm{g}$ of the purified P37 protein. Protein emulsification and immunization were performed as previously described [30]. P37 protein emulsified with complete Freund's adjuvant (Sigma-Aldrich) was injected subcutaneously. After an interval of 3 weeks, the mice were boosted with P37 protein emulsified in incomplete Freund's adjuvant (Sigma-Aldrich). After a 2-week interval, mice were intraperitoneally administered $80 \mu \mathrm{g}$ of P37 protein without adjuvant. On the $3 \mathrm{~d}$ of intraperitoneal immunization, the mice were euthanized by cervical dislocation (disconnecting the spinal cord from the brain, causing the experimental animals to die without pain), and their splenocytes were fused with SP2/0 cells as previously described [31]. The fused cells were mixed with DMEM medium containing hypoxanthineaminopurine-thymidine (HAT) (Sigma-Aldrich, New York, NY, USA) and 20\% FBS. Cells were cultured together and the media were replaced with selection medium containing hypoxanthine-thymidine (HT) (Sigma-Aldrich) and 10\% FBS after $5 \mathrm{~d}$ [25]. Hybridoma supernatants were collected after $7 \mathrm{~d}$ and screened for the presence of Mhr-specific antibodies by indirect enzyme-linked immunosorbent assay (ELISA) [28]. The purified P37 protein was used as the coating antigen at a concentration of $5 \mathrm{ng} / \mu \mathrm{L}$ as previously described [28]. Positive hybridoma cells were cloned three times by limiting dilution and stored in liquid nitrogen.

\section{Characterization of mAbs against P37 protein}

The subtypes of mAbs produced were determined using the SBA Clonotyping System-HRP kit (Southern Biotech, Birmingham, AL, USA) according to the manufacturer's instructions. The hybridoma supernatant was added as a primary antibody and horseradish peroxidase (HRP)-conjugated goat anti-mouse antibody was used as the secondary antibody. Color development and screening were performed as described above. The reactivity of mAbs to Mhr was determined by Western blot analysis as described above and Mhr-specific antigen in the lung tissue of Mhr-infected pigs was detected using immunohistochemical assays. The proteolytic antigen retrieval step of the immunohistochemical assay was modified to include proteinase K (Merck Life Science Co. Ltd., Shanghai, China). Uninfected lung tissue (by PCR detection) was used as the negative control and the cross reactivity of mAbs to Mhp was tested by immunohistochemistry using Mhp positive tissues.

\section{Preliminary identification of P37 protein B cell line epitopes using P37-specific mAbs}

To identify the epitopes of the mAbs produced against the P37 protein, a series of nucleotide sequences encoding aa regions of P37 were cloned into the $B a m \mathrm{H} \mathrm{I}$ and Xho I sites of pGEX-6P-1 (Fig. 9). After sequencing, E. coli BL21 cells were transformed with the recombinant plasmids, which were induced by the addition of $1 \mathrm{mM}$ isopropyl $\beta$ D-1-thiogalactopyranoside (IPTG) and incubated at $37^{\circ} \mathrm{C}$ with shaking for $6 \mathrm{~h}$. Cultures were harvested and lysed, and lysates were analyzed by SDS-PAGE and Western blot. Lysate from induced pGEX-6p-1 E. coli BL-21 cells was used as the negative control. The prepared mAbs against the P37 protein were used as the primary antibody, and DyLight ${ }^{\mathrm{mi}}$ 680-Labeled Antibody to Mouse IgG (H + L) $(1: 10,000)$ was used as the secondary antibody. The plate was incubated for $1 \mathrm{~h}$ at room temperature in the dark, washed three times with PBST, and analyzed using the Odyssey infrared imaging system (Licor Odyssey). 


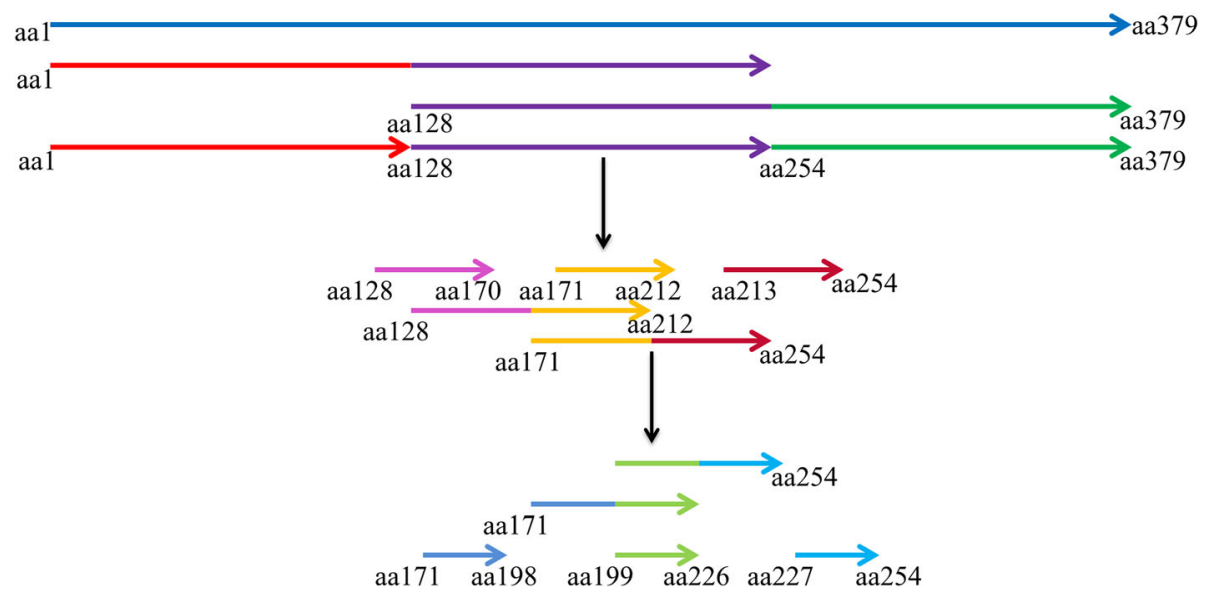

Fig. 9 P37 protein fragment truncation protocol. Schematic representation of the protocol for P37 protein epitope identification by fragment truncation. The black arrow represents regions (aa128-254, aa171-254, and aa199-226) that reacted with the four prepared mAbs

\section{Precise localization of the P37 protein B cell epitope}

To define the minimal linear epitope of the P37 protein, aa199-226 were deleted one by one (listed in Table 2) and their corresponding peptides were assessed by Western blot as described above. The immunoreactivity of the core epitope to corresponding mAbs was then analyzed.

\section{Multiple alignment of P37 amino acid sequences}

Multiple alignments of aa sequences of the P37 protein of seven Mhr isolates (GenBank accession Nos. CP002170.1, CP002669.1, CP003231.1, CP016817.1, NC_019552.1, NC_ 022807.1, and NZ_LS991950.1) were performed using the

Table 2 Peptides used in this study and their corresponding amino acid sequences

\begin{tabular}{ll}
\hline Peptide & Peptide sequence \\
\hline B1 (aa200-226) & NDETLAKIKKAWNDKDWNTFRNFGILH \\
B2 (aa201-226) & DETLAKIKKAWNDKDWNTFRNFGILH \\
B3 (aa202-226) & ETLAKIKKAWNDKDWNTFRNFGILH \\
B4 (aa203-226) & TLAKIKKAWNDKDWNTFRNFGILH \\
B5 (aa204-226) & LAKIKKAWNDKDWNTFRNFGILH \\
B6 (aa205-226) & AKIKKAWNDKDWNTFRNFGILH \\
B7 (aa206-226) & KIKKAWNDKDWNTFRNFGILH \\
B8 (aa207-226) & IKKAWNDKDWNTFRNFGILH \\
B9 (aa199-225) & GNDETLAKIKKAWNDKDWNTFRNFGIL \\
B10 (aa199-224) & GNDETLAKIKKAWNDKDWNTFRNFGI \\
B11 (aa199-223) & GNDETLAKIKKAWNDKDWNTFRNFG \\
B12 (aa199-222) & GNDETLAKIKKAWNDKDWNTFRNF \\
B13 (aa199-221) & GNDETLAKIKKAWNDKDWNTFRN \\
B14 (aa199-220) & GNDETLAKIKKAWNDKDWNTFR \\
B15 (aa199-219) & GNDETLAKIKKAWNDKDWNTF \\
B16 (aa199-218) & GNDETLAKIKKAWNDKDWNT \\
\hline
\end{tabular}

Clustal W method within DNASTAR software version 7.0 (https://www.dnastar.com/software/).

\section{Homology modeling of P37 epitopes}

Homology modeling of aa1-379 of the P37 protein was performed using SWISS-MODEL (https://www.swissmodel.expasy.org/interactive). The spatial locations of the identified P37 protein epitopes were determined by mapping the epitopes to a three-dimensional model of the P37 protein using Chimera 1.11.2 software (https://www. cgl.ucsf.edu/chimera/).

\section{Abbreviations}

aa: amino acid; CCU: color change unit; DMEM: Dulbecco's modified Eagle's medium; ELISA: enzyme-linked immunosorbent assay; FBS: fetal bovine serum; HRP: horseradish peroxidase; HT: hypoxanthine-thymidine; IFA: indirect immunofluorescence assays; IgG: immunoglobulin G; IPTG: isopropyl $\beta$-D-1-thiogalactopyranoside; kDa: kilodalton; Lys: Iysine; mAbs: monoclonal antibodies; Mhr: Mycoplasma hyorhinis; MOI: multiplicity of infection; PBS: phosphate buffered saline; PBST: PBS containing Tween 20; HAT hypoxanthine-aminopurine-thymidine; PCR: polymerase chain reaction; PFUs: plaque forming units; Phe: phenylalanine; SDS-PAGE: sodium dodecyl sulfate-polyacrylamide gel electrophoresis; sf21: Spodoptera frugiperda; K: kappa

\section{Acknowledgments}

Not applicable.

\section{Authors' contributions}

HZZ and YWW contributed equally to this work, carrying out all studies, participating in study design, and drafting the manuscript. CML and LF conceived and designed the experiments and analyzed the results. $L P H, D L$, YXX, DLX, and HQB performed the experiments. All authors read and approved the final version of the manuscript.

\section{Funding}

This work was supported by a New Technology for Prevention and Control of Important Diseases of Pigs grant (2017YFD0500602). The funding body was not involved in study design, data collection, analysis and in writing the manuscript. 


\section{Availability of data and materials}

The datasets used and/or analyzed during the current study are available from the corresponding author on reasonable request. All data generated or analyzed during this study are included in this published article.

\section{Ethics approval and consent to participate}

The experimental procedures used in this study were approved by the Animal Ethics Committee of the Harbin Veterinary Research Institute of the Chinese Academy of Agricultural Sciences (Heilongjiang-SYXK-2017-009) and were performed in accordance with the relevant animal ethics principles.

\section{Consent for publication}

Not applicable.

\section{Competing interests}

The authors declare that they have no competing interests.

\section{Author details}

'Division of Swine Digestive System Infectious Diseases, State Key Laboratory of Veterinary Biotechnology, Harbin Veterinary Research Institute, Chinese Academy of Agricultural Sciences, No. 678 Ha-ping Street, Xiang-fang region, Harbin 150069, China. ${ }^{2}$ College of Veterinary Medicine, Ji Lin University, Changchun 130062, China.

Received: 1 May 2019 Accepted: 16 October 2019

Published online: 05 November 2019

\section{References}

1. Carter CR, McKay KA. A Pleuropneumonia-like organism associated with infectious atrophic rhinitis of swine. Can J Comp Med Vet Sci. 1953;17(10):413-6.

2. Lee JA, Oh YR, Hwang MA, Lee JB, Park SY, Song CS, Choi IS, Lee SW. Mycoplasma hyorhinis is a potential pathogen of porcine respiratory disease complex that aggravates pneumonia caused by porcine reproductive and respiratory syndrome virus. Vet Immunol Immunopathol. 2016;177:48-51.

3. Lin JH, Chen SP, Yeh KS, Weng CN. Mycoplasma hyorhinis in Taiwan: diagnosis and isolation of swine pneumonia pathogen. Vet Microbiol. 2006; 115(1-3):111-6.

4. Martinson B, Minion FC, Kroll J, Hermann J. Age susceptibility of caesarian derived colostrum deprived pigs to mycoplasma hyorhinis challenge. Vet Microbiol. 2017;210:147-52.

5. Clavijo MJ, Murray D, Oliveira S, Rovira A. Infection dynamics of mycoplasma hyorhinis in three commercial pig populations. Vet Rec. 2017;181(3):68.

6. Amass SF, Clark LK, van Alstine WG, Bowersock TL, Murphy DA, Knox KE, Albregts SR. Interaction of mycoplasma hyopneumoniae and Pasteurella multocida infections in swine. J Am Vet Med Assoc. 1994;204(1):102-7.

7. Petersen AC, Oneal DC, Seibel JR, Poel K, Daum CL, Djordjevic SP, Minion FC. Cross reactivity among the swine mycoplasmas as identified by protein microarray. Vet Microbiol. 2016;192:204-12.

8. Beko K, Felde O, Sulyok KM, Kreizinger Z, Hrivnak V, Kiss K, Biksi I, Jerzsele A, Gyuranecz M. Antibiotic susceptibility profiles of mycoplasma hyorhinis strains isolated from swine in Hungary. Vet Microbiol. 2019;228:196-201.

9. Yang H, Qu L, Ma H, Chen L, Liu W, Liu C, Meng L, Wu J, Shou C. Mycoplasma hyorhinis infection in gastric carcinoma and its effects on the malignant phenotypes of gastric cancer cells. BMC Gastroenterol. 2010;10:132.

10. Boyarskikh UA, Shadrina AS, Smetanina MA, Tsepilov YA, Oscorbin IP, Kozlov W, Kel AE, Filipenko ML. Mycoplasma hyorhinis reduces sensitivity of human lung carcinoma cells to Nutlin-3 and promotes their malignant phenotype. J Cancer Res Clin Oncol. 2018;144(7):1289-300.

11. Kawashima K, Katsuda K, Tsunemitsu H. Epidemiological investigation of the prevalence and features of postweaning multisystemic wasting syndrome in Japan. J Vet Diagn Investig. 2007;19(1):60-8.

12. Palzer A, Haedke K, Heinritzi K, Zoels S, Ladinig A, Ritzmann M. Associations among Haemophilus parasuis, mycoplasma hyorhinis, and porcine reproductive and respiratory syndrome virus infections in pigs with polyserositis. Can Vet J. 2015;56(3):285-7.

13. Bai F, Ni B, Liu M, Feng Z, Xiong Q, Shao G. Mycoplasma hyopneumoniae-derived lipid-associated membrane proteins induce inflammation and apoptosis in porcine peripheral blood mononuclear cells in vitro. Vet Microbiol. 2015;175(1):58-67.
14. Yogev D, Rosengarten R, Watson-McKown R, Wise KS. Molecular basis of mycoplasma surface antigenic variation: a novel set of divergent genes undergo spontaneous mutation of periodic coding regions and $5^{\prime}$ regulatory sequences. EMBO J. 1991;10(13):4069-79.

15. Dudler R, Schmidhauser C, Parish RW, Wettenhall RE, Schmidt T. A mycoplasma high-affinity transport system and the in vitro invasiveness of mouse sarcoma cells. EMBO J. 1988;7(12):3963-70.

16. Liu WB, Zhang JZ, Jiang BH, Ren TT, Gong MM, Meng L, Shou CC. Lipoprotein p37 from mycoplasma hyorhinis inhibiting mammalian cell adhesion. J Biomed Sci. 2006;13(3):323-31.

17. Ketcham CM, Anai S, Reutzel R, Sheng S, Schuster SM, Brenes RB, AgbandjeMcKenna M, McKenna R, Rosser CJ, Boehlein SK. p37 induces tumor invasiveness. Mol Cancer Ther. 2005;4(7):1031-8.

18. Urbanek C, Goodison S, Chang M, Porvasnik S, Sakamoto N, Li CZ, Boehlein SK, Rosser CJ. Detection of antibodies directed at M. hyorhinis p37 in the serum of men with newly diagnosed prostate cancer. BMC Cancer. 2011;11:233.

19. Bumgardner EA, Bey RF, Lawrence PK. A p37-based ELISA used to monitor anti-mycoplasma hyorhinis IgG in serum from pigs immunized with inactivated M-hyorhinis vaccines. J Vet Diagn Investig. 2018;30(5):755-9.

20. Palzer A, Kolb K, Strutzberg-Minder K, Zoels S, Eddicks M, Heinritzi K, Ritzmann M. Serological course investigations of Haemophilus parasuis and mycoplasma hyorhinis in three pig farms. Schweiz Arch Tierheilkd. 2015; 157(2):97-103.

21. Magnusson U, Wilkie B, Mallard B, Rosendal S, Kennedy B. Mycoplasma hyorhinis infection of pigs selectively bred for high and low immune response. Vet Immunol Immunopathol. 1998;61(1):83-96.

22. Morita T, Fukuda H, Awakura T, Shimada A, Umemura T, Kazama S, Yagihashi T. Demonstration of mycoplasma hyorhinis as a possible primary pathogen for porcine otitis media. Vet Pathol. 1995;32(2):107-11.

23. Jayagopala Reddy NR, Wilkie BN, Borgs P, Mallard BA. Cytokines in mycoplasma hyorhinis-induced arthritis in pigs bred selectively for high and low immune responses. Infect Immun. 2000;68(3):1150-5.

24. Sippel KH, Robbins AH, Reutzel R, Boehlein SK, Namiki K, Goodison S, Agbandje-McKenna M, Rosser CJ, McKenna R. Structural insights into the extracytoplasmic thiamine-binding lipoprotein p37 of mycoplasma hyorhinis. J Bacteriol. 2009;191(8):2585-92.

25. Sun J, Huang L, Wei Y, Wang Y, Chen D, Du W, Wu H, Feng L, Liu C. Identification of three PPV1 VP2 protein-specific B cell linear epitopes using monoclonal antibodies against baculovirus-expressed recombinant VP2 protein. Appl Microbiol Biotechnol. 2015;99(21):9025-36.

26. Liu D, Hu J, Dong H, Huang L, Wei Y, Xia D, Zhu H, Wang X, Wu H, Wang X, et al. Identification of three linear $B$ cell epitopes using monoclonal antibodies against bovine enterovirus VP2 protein. Appl Microbiol Biotechnol. 2019;103(18):7467-80.

27. Gomes Neto JC, Strait EL, Raymond M, Ramirez A, Minion FC. Antibody responses of swine following infection with mycoplasma hyopneumoniae, M. hyorhinis, M. hyosynoviae and M. flocculare. Vet Microbiol. 2014;174(1-2):163-71.

28. Chen D, Wei Y, Huang L, Wang Y, Du W, Sun J, Wu H, Feng L, Liu C. Characterization and application of monoclonal antibodies against mycoplasma hyorhinis pyruvate dehydrogenase E1 complex subunit alpha. Appl Microbiol Biotechnol. 2016;100(8):3587-97.

29. Chen D, Wei Y, Huang L, Wang Y, Sun J, Du W, Wu H, Liu C. Synergistic pathogenicity in sequential coinfection with mycoplasma hyorhinis and porcine circovirus type 2. Vet Microbiol. 2016;182:123-30.

30. Huang L, Lu Y, Wei Y, Guo L, Liu C. Development of a blocking ELISA for detection of serum neutralizing antibodies against porcine circovirus type 2 . J Virol Methods. 2011;171(1):26-33.

31. Galfre G, Milstein C: Preparation of monoclonal antibodies: strategies and procedures. Methods Enzymol. 1981; 73(Pt B):3-46.

\section{Publisher's Note}

Springer Nature remains neutral with regard to jurisdictional claims in published maps and institutional affiliations. 